\title{
Landscape connectivity and predator-prey population dynamics
}

\author{
Jacopo A. Baggio · Kehinde Salau • \\ Marco A. Janssen • Michael L. Schoon • \\ Örjan Bodin
}

Received: 21 July 2009/Accepted: 27 May 2010/Published online: 10 June 2010

(C) Springer Science+Business Media B.V. 2010

\begin{abstract}
Landscapes are increasingly fragmented, and conservation programs have started to look at network approaches for maintaining populations at a larger scale. We present an agent-based model of predator-prey dynamics where the agents (i.e. the individuals of either the predator or prey population) are able to move between different patches in a landscaped network. We then analyze population level and coexistence probability given node-centrality measures that characterize specific patches. We show that both predator and prey species benefit from
\end{abstract}

Electronic supplementary material The online version of this article (doi:10.1007/s10980-010-9493-y) contains supplementary material, which is available to authorized users.

\section{J. A. Baggio $(\varangle)$}

School of International Development, University of East Anglia, NR47TJ Norwich, UK

e-mail: j.baggio@uea.ac.uk

J. A. Baggio - K. Salau · M. A. Janssen - M. L. Schoon Center for the Study of Institutional Diversity, School of Human Evolution and Social Change, Arizona State University, Tempe, AZ 85287-2402, USA

K. Salau

Mathematical, Computational and Modeling Science Center, Arizona State University, Tempe, AZ 85287 2402, USA

Ö. Bodin

Stockholm Resilience Center, Stockholm University, 10691 Stockholm, Sweden living in globally well-connected patches (i.e. with high closeness centrality). However, the maximum number of prey species is reached, on average, at lower closeness centrality levels than for predator species. Hence, prey species benefit from constraints imposed on species movement in fragmented landscapes since they can reproduce with a lesser risk of predation, and their need for using anti-predatory strategies decreases.

Keywords Networks - Landscape · Predator-prey $\cdot$ Coexistence $\cdot$ Survival probabilities . ABM $\cdot$ IBM

\section{Introduction}

Species are distributed heterogeneously and, given the variety of habitats that exist in nature, habitat fragmentation per se does not necessarily threaten many species. However, in recent years, fragmentation seems to have conspicuously accelerated given human population growth and urban sprawl, and the pace and scale of fragmentation is increasingly threatening for species' persistence. Hence, understanding the ecological consequences of habitat fragmentation is now part of most research agendas that deal with conservation, biodiversity and adaptation to climatic change. More recently we have seen the increasing use of a network approach in conservation of both marine and terrestrial landscapes (e.g. 
Bodin and Norberg 2007; Planesa et al. 2009; Urban et al. 2009). This network approach describes landscapes as networks of habitat patches (nodes) connected by edges representing links between different patches (Urban and Keitt 2001), indicating the ability of an organism (predator or prey) to directly disperse/diffuse from one patch to another (from node $i$ to node $j$ ) (Pascual-Hortal and Saura 2006). Movement of organisms to/from a specific patch is limited to connected habitat patches close enough to allow for species migration (Bodin and Norberg 2007). Therefore, a network perspective allows us to combine landscape patterns and metapopulation dynamics (Urban and Keitt 2001; Bodin and Norberg 2007; Minor and Urban 2007) to better understand the influence of fragmentation on population persistence. However, almost all of these types of studies have focused on single species and how they might be affected by various levels of habitat fragmentation. Here, we will focus on how habitat fragmentation may affect two interacting species (a predator and a prey species).

Issues of space and time-scale increase the complexity of predator-prey dynamics as their inclusion leads to substantially different outcomes even if all other variables that affect these dynamics are kept constant (Fahrig and Nuttle 2005). Past literature has dealt in depth with land-fragmentation and its effect on movement of species (Rougharden 1977, 1978; Droz and Pekalski 2001; Bolker 2003; Fahrig and Nuttle 2005; Pascual-Hortal and Saura 2006; Nonaka and Holme 2007; Inchausti and Ballesteros 2008). Thus, in understanding the importance of spatial heterogeneity it is necessary to focus on how fragmentation affects population levels and the likelihood of predator-prey coexistence. We aim to uncover the significance of heterogeneous fragmentation of landscapes, by representing them as networks and looking at the relationship that exists between predator-prey dynamics and network metrics (e.g. node-centrality). In other words, we seek to understand how the structure of habitat fragmentation affects predator-prey dynamics and the probability of coexistence.

Fragmentation, connectivity, and spatial conditions need to be taken into account when dealing with predator-prey population dynamics as species diffuse and respond to landscape patterns and the surrounding environment (Rougharden 1977, 1978). According to previous experimental papers, the landscape structure is able to alter predation pressure (With et al. 2002), thus modifying prey development depending on the landscape structural changes (Kareiva 1987). Landscape heterogeneity per se does not seem to have any significant effect on predator-prey dynamics. However, when combined with movement capabilities, it may lead to important alterations of predators and prey populations (Fahrig 1998).

The interaction between predators and prey has been studied with differential equations (i.e. LotkaVolterra), reaction-diffusion equations (McLaughlin and Roughgarden 1991; Benson et al. 1993), and individual based models (Cuddington and Yodzis 2000; Droz and Pekalski 2001; DeAngelis and Mooij 2005; Hovel and Regan 2008). While in analytic models population is treated as a whole (homogeneous mixing), individual (agent) based models (IBM or ABM) centre on individual differences (DeAngelis and Mooij 2005; Grimm and Railsback 2005; Breckling et al. 2006). ABMs allow population dynamics to emerge from individual predators and prey, and we believe this approach to be essential if we are to uncover the complexities arising in predator-prey systems on heterogeneous and fragmented landscapes (McCauley et al. 1993).

This paper focuses on the consequences of movement between patches, rather than spatial details of a single patch, by modelling individual predator and prey on heterogeneous landscapes, which we model as networks of habitat patches. The central research questions are as follows:

- How does the underlying network of habitat patches influence population levels?

- Does network connectivity and centrality of a patch drive predator-prey dynamics and the probability of coexistence?

\section{Methods}

On a given landscape, we model predator-prey interaction according to the $\mathrm{ABM}$ proposed by Wilson (1998), whose work aimed at linking two different modelling frameworks, ABMs and coupled differential equations. We extend this study by including a networked landscape where nodes represent habitat patches. Habitat patches are considered land where prey can eat, predators can hunt, and both 
species can reproduce (Ives and Dobson 1987; Droz and Pekalski 2001). Edges represent movement possibilities between different patches. In the past, species models of networks with small and large patch numbers have been studied. A small number of patches have been studied to resemble ecosystems, while a larger number have been used to examine multiple species coexistence (Cominsa and Hassell 1996; Blasius et al. 1999; Hastings 2001; Jansen 2001). More recently Holland and Hastings (2008) have developed a manageable ten-patch model that supplements the realism of models with small number of patches while displaying results similar to networks with a larger number of habitat patches.

Given the aim of this model, we rely heavily on the movement capabilities of agents between different habitat patches. Recently, movement of predators and prey has been widely researched in order to explicitly incorporate space into modelling species interaction with emphasis on predators' searching strategies and the anti-predatory behaviour of prey (McLaughlin and Roughgarden 1991; Linhares 1999; Inchausti and Ballesteros 2008). In the model presented, agents actively choose to move according to inputs given by the surrounding environment. The results of the model presented here, where a threshold rule is used to determine the movement decisions by prey and predators, can be compared to more simplistic models of migration based on diffusive and random movement. This comparison may shed further light on the importance of understanding the effects of landscape connectivity. Nonetheless, few studies take active behavior into account and we think that species do move according to the feedback they receive from the surrounding environment (e.g. intra-species competition, search strategies and antipredatory behavior).

A detailed description of the ABM proposed is provided in the ODD (Overview, Design concepts, and Details) protocol available in the supplementary material. The ODD protocol is a standard protocol for describing individual and agent based models (Grimm et al. 2006), so as to allow for a deeper understanding of the model and to facilitate replication.

The network of habitat patches

To build on the work of Holland and Hastings (2008), we consider a landscape with $N=10$ habitat patches and vary the number of edges $(E)$ that connect them. Habitat patches (nodes) are placed randomly on a two-dimensional grid and are connected through edges according to their proximity to other patches (patches within small Euclidean distance from each other are connected first) as shown in Fig. 1. Geographic proximity is an important aspect concerning the network topology of a landscape (Minor and Urban 2007, 2008); the networked landscape presented is based on Euclidean distances in order to better simulate movement of species on real landscapes. We do not allow loops and multiple edges in our network. All habitat patches are considered equal; as a result, the ability to sustain prey does not vary throughout the patches. We interpret the landscape as an undirected, un-weighted network. An undirected network contains edges that enable movement from one node to another and vice versa. An un-weighted network only considers edges as connectors and not pathways with explicit distance (note, Euclidean distance is only used in order to determine if a connection, an edge, should exist between two patches). Nonetheless the probability of movement may still be asymmetric depending on the strength of a node's centrality. For instance, agents on node $i$ may only be permitted to move towards node $j$ while agents on node $j$ have the possibility to move to many different nodes, including node $i$ (e.g. Fig. $1 \mathrm{~b}$ bottom right node and its neighbour). Although we are aware that a more complex network representation may lead to different results, an undirected, un-weighted network is still able to provide information enabling the assessment of patch importance on the species' movement abilities (Estrada and Bodin 2008).

\section{The species}

There are two types of agent-sets, predator and prey, each of which is assigned randomly to a habitat patch. In our mathematical notation we use subscript 1 for prey and 2 for predators. The initial number of predators and prey is proportional to the number of nodes in the network, as shown in Table 1. Each time-step prey have the ability to reproduce with probability $P_{r, 1}$ and to die via predation with probability $P_{k, 2}$, if both prey and predator are assigned to the same patch $i$. Predators may also reproduce at every time-step with probability $P_{r, 2}$, given they have successfully attacked (thus killed) a 
Fig. 1 Graphical representation of a geoproximity network for $E=5(\mathbf{a}), E=15$ (b), $E=20(\mathbf{c})$ and $E=25(\mathbf{d})$, $E=30(\mathbf{e}), E=35(\mathbf{f})$, and $E=45$ (g)
Table 1 Summary of variables, symbols and values used in the ABM
A

A

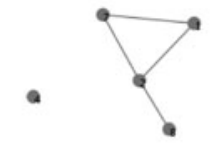

D

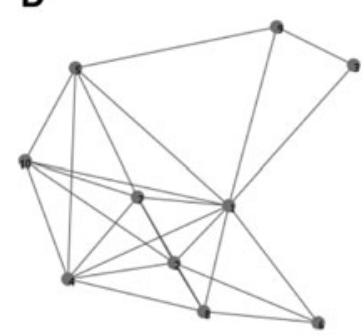

B

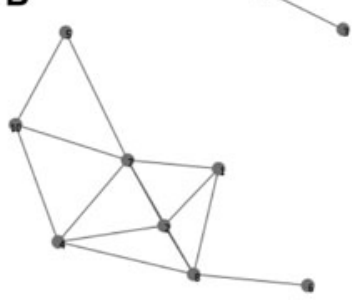

E
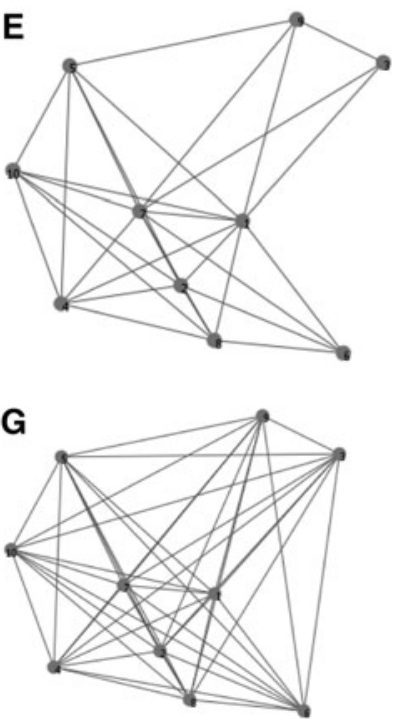

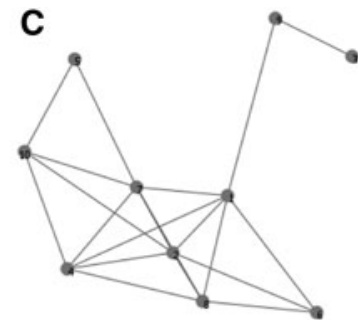

$\mathbf{F}$

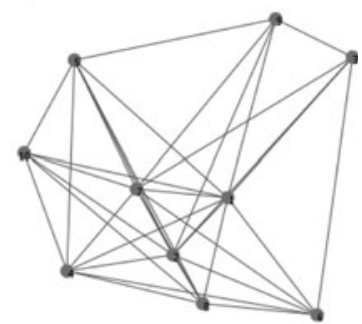

\begin{tabular}{lll}
\hline Simbol & Variable name & $\begin{array}{l}\text { Values from distributions used } \\
\text { in Monte Carlo simulations }\end{array}$ \\
\hline$N$ & Number of nodes & 10 \\
$E$ & Number of edges & Varies from 0 to 45 \\
$C$ & Size of a node & 100 \\
$n_{1}$ & Initial number of prey & Poisson with mean $25 * N$ \\
$P_{r, 1}$ & Prey reproduction rate & Poisson with mean $0.25(25 \%)$ \\
$D_{U, 1}$ & Prey density upper limit & Random uniform distribution $[0.5,0.9]$ \\
$D_{L, 1}$ & Prey density lower limit & Random uniform distribution $[0.2,0.4]$ \\
$n_{2}$ & Initial number of predators & Poisson with mean $10 * N$ \\
$P_{r, 2}$ & Predator reproduction rate & Poisson with mean $0.2(20 \%)$ \\
$P_{k, 2}$ & Predation probability & Poisson with mean $0.2(20 \%)$ \\
$P_{m, 2}$ & Predator death rate & Poisson with mean $0.06(6 \%)$ \\
$D_{U, 2}$ & Predator density upper limit & Random uniform distribution $[0.3,0.6]$ \\
$T_{h}$ & Predator handling time & 3 \\
\hline & &
\end{tabular}

prey and are currently in their handling period $\left(T_{h}\right)$, a timeframe in which predators are consuming the prey and hence have the "energy" to reproduce. Predators die naturally according to a fixed death rate $\left(P_{m, 2}\right)$.
Drawing from the literature we simplify the movement behaviour of agents between patches. Agents move between patches according to density thresholds in one or both species. Prey move if their density 
in a given patch $i\left(D n_{l i}\right)$ is higher than a predetermined threshold $\left(D_{U, 1}\right)$ so as to mimic intra-species competition for food. Prey also move if the predator density $\left(D n_{2 i}\right)$ rises above a predetermined threshold $\left(D_{U, 2}\right)$ in order to mimic anti-predator behaviour (Ives and Dobson 1987; Luttberg and Schmitz 2000; Lima 2002; Nelson et al. 2004; Creel et al. 2005; Fischhoff et al. 2007). If prey density falls below a predetermined threshold $\left(D_{L, 1}\right)$, predators move between patches looking for higher densities of prey, thus imitating predatory search strategies (Linhares 1999; Lima 2002; Ioannou et al. 2008). Table 1 summarizes variables and values used in the ABM model.

Parameters are set at the beginning of each run as described in Table 1. Internal species parameters are drawn from the distributions described, while the number of edges, $E$, varies from 0 to 45 as shown in Fig. 1. We measure the population levels for both predators and prey individually for every patch throughout the different simulation runs. The mean values of the parameters presented in Table 1 are one of the configurations that enable coexistence in the model presented by Wilson (1998). We use Monte Carlo methods to explore a wider parameter space to test the sensitivity of the outcomes. By altering the number of existing connections between patches we show the importance of landscape fragmentation in the welfare of predator-prey systems independently from species reproduction, death and predation rates.

Network structures and predator-prey dynamics

The analysis is based on network metrics that statistically characterize landscape connectivity. In what follows we briefly describe the metrics used to uncover the relationships existing between patchcentrality, landscape connectivity and population dynamics; however, for in depth information on the metrics used, please refer to Boccaletti et al. (2006), da Fontoura Costa et al. (2007). We focus our analysis on node-centrality and other network metrics that are relevant for measuring the dispersal of species. Detailed results for each metric used can be found in the supplemental material. Here we concentrate on two node-centrality measures and three network metrics that are most important when assessing coexistence probabilities and shaping predator-prey population levels. Therefore we concentrate on closeness centrality (clos), an average of node-centrality measures (closeness centrality, node degree and local efficiency) denoted cde, network average local efficiency (avgel), global efficiency (avgeg), and the percentage of nodes belonging to the giant connected component ( $\mathrm{gcc}$ ). Closeness centrality measures the average geodesic distance (shortest path length) between one node and all other nodes in the network within its reach. In other words, a node is globally central if it is reachable from many other nodes. Local efficiency is the average efficiency of local sub-graphs (Latora and Marchiori 2001). In essence, it is a measure of how effectively information spreads through a network on a local scale; in this case, the information is perceived as species diffusion between connected patches. Global efficiency is defined as the average of the inverse distance between two nodes; it is related to an agent's movement ability and it is measurable for unconnected graphs (Latora and Marchiori 2001; Crucitti et al. 2004). The giant connected component can be defined as the largest part of the network whose nodes are connected to each other. All the measures used are normalized to ease comparisons between the different networks created through the simulations.

\section{Results}

We utilize Monte Carlo method to gain a broader understanding of the model dynamics. We vary the number of edges from 0 to 45 (where a simulation with 45 edges represents a fully connected network). For a given number of edges, 1000 simulations with different parameter configurations drawn from the distributions presented in Table 1 are performed. Each simulation lasts for 5000 time steps. Since our main focus is on long-term population dynamics and the probability of species coexistence, we collect the average population levels of predators and prey from the last 1000 time-steps.

Using Monte Carlo method allows us to assess the importance of node centrality and other network metrics under a wide range of dynamics. Figure 2 displays the dynamics of 8 select runs from the original ten thousand. These runs were specifically chosen because they represent the distinct regimes and population patterns that arise from the simulations. Figure $2 \mathrm{a}$ focuses on population level of the whole network while $2 \mathrm{~B}$ and $2 \mathrm{C}$ focus on population 
at the node level, our local representatives being nodes 1 and 5, respectively (node 1 and 5 have been chosen as representative of local interaction, graphs of the dynamics on all the nodes for all the runs are presented in the supplementary material). Note the differences between global and local dynamics. Species can abandon a certain node for some period of time due to intra- and/or interspecies competition (i.e. prey on node 1 at run 1) but may persist on other connected nodes, thus fostering global survival (i.e. total prey on the network during run 1). Moreover, if nodes are connected, temporary extinction on a node is also possible, as shown in Fig. 3d. The greater fluctuation of both species that occur on a local scale, compared with what happens to population trends at the network level, is due to migration, a local phenomenon (e.g. Fig. $2 \mathrm{a}$ and $\mathrm{b}$ for run 7, and Fig. $3 a$ and $b$ ). Figure 3 displays selected runs from
Fig. 2 with shorter time intervals for magnified viewing of some of these dynamics.

Table 2 contains the internal species parameters used, the corresponding values for the metrics of the whole network, while Table 3 contains the centrality measures for nodes, 1 and 5, for the 8 representative runs. Figure 2 and Tables 2 and 3 show, in general, that a positive correlation exists between connectivity level and long-term coexistence; as we progress from run 1 to 8 , the number of edges increase and so does the possibility for coexistence. However, between runs 4 and 6 , where the network contains 30 to 35 edges, the predator population becomes variable. In run 4 (30 edges), the predators are able to coexist while they fall victim to early extinction in runs 5 (30 edges) and 6 ( 35 edges). All three intermediate runs consist of similar network metrics (avgeg of 0.833 , 0.826 , and 0.889 respectively), and so, the reason for

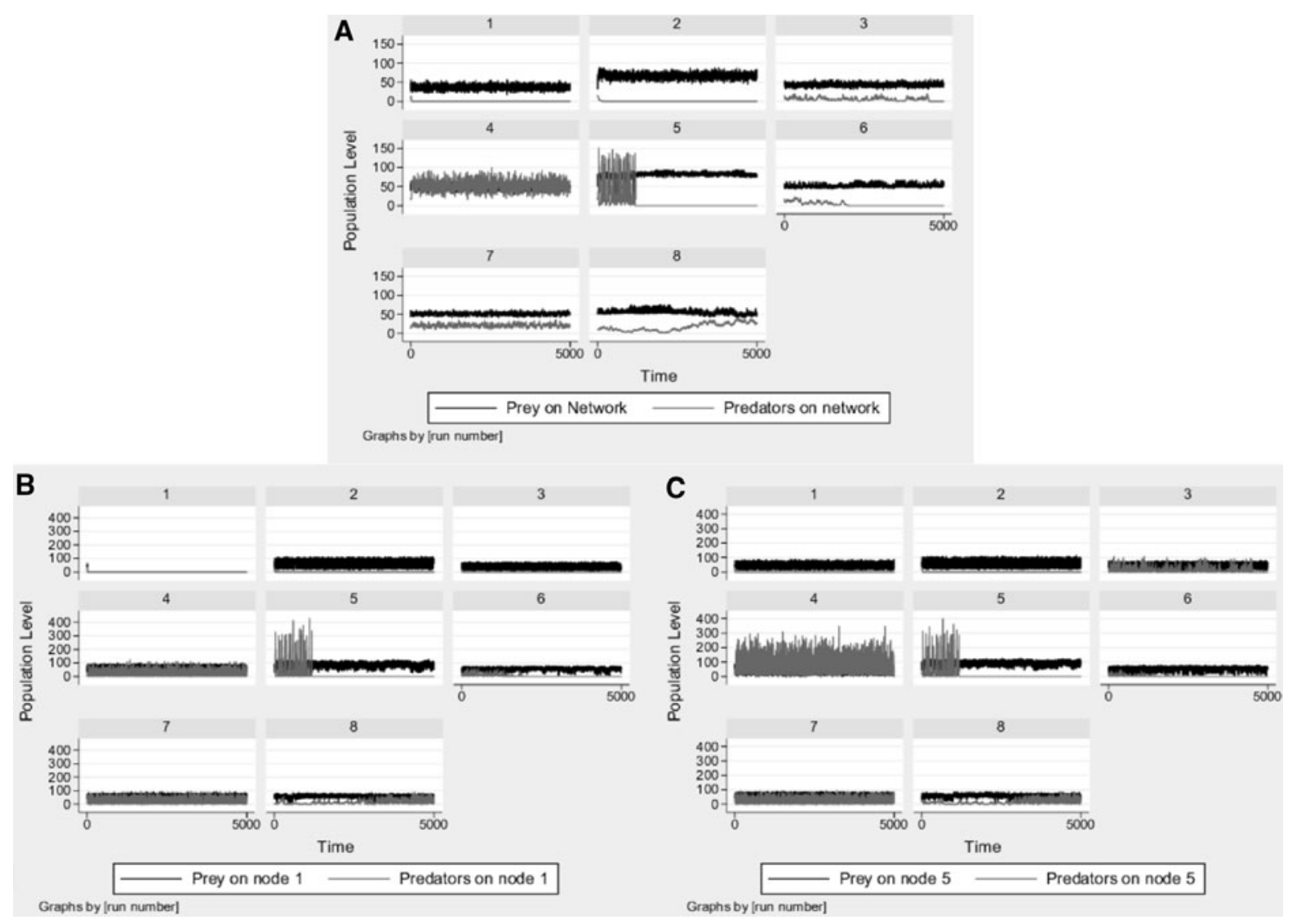

Fig. 2 Dynamics of the model for 8 select runs. These runs represent the total regime of dynamics under the parameter space explored. Global (network) dynamics (a) and local (node) dynamics (b, c). Parameter values, network and node centralities are presented in Tables 2 and 3. Network population levels are divided by 10 

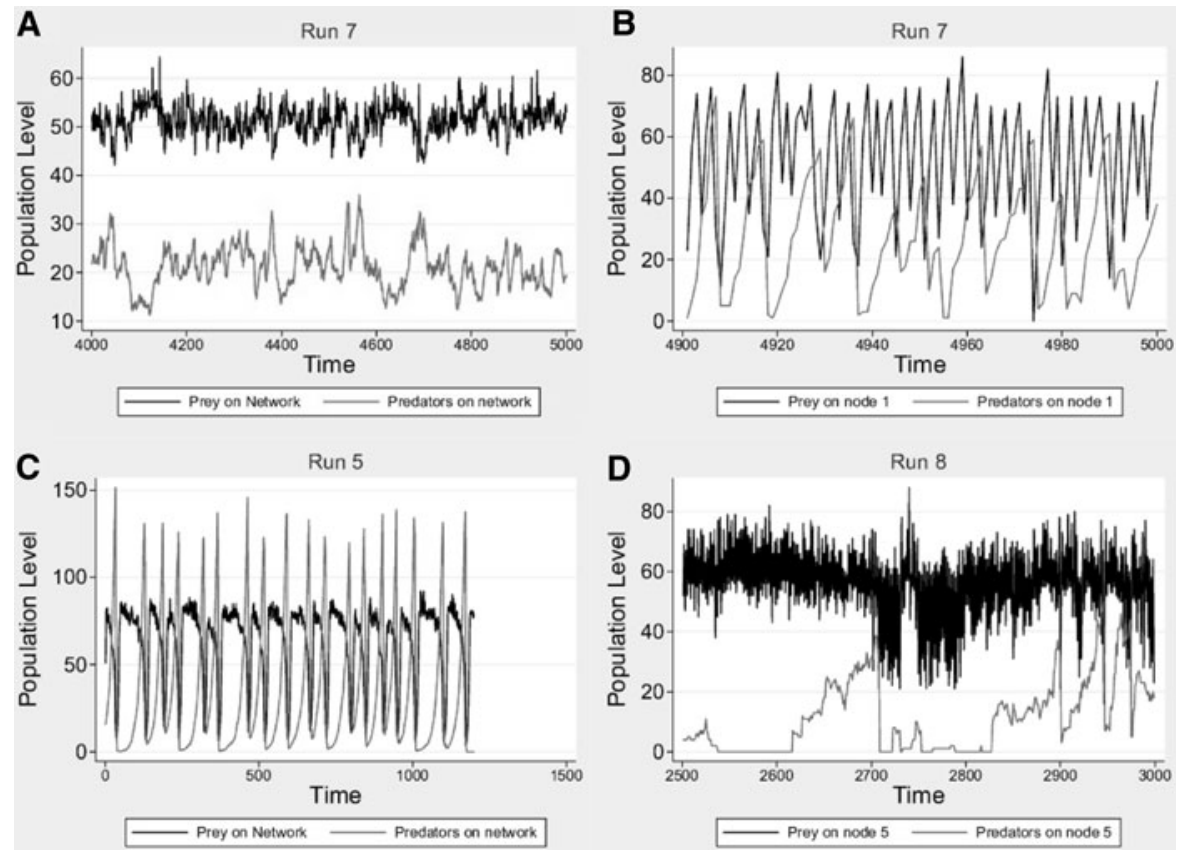

Fig. 3 Dynamics of the model for select runs, from Fig. 2, on shorter time intervals (to magnify visuals). a and b represent run 7 at the global and at the local scale (network vs node 1), where local oscillations are more amplified. Differences in the time-scale used are necessary in order to clearly visualize the

patterns. c represent a magnified visual of the high fluctuation that occur in run 5, while $\mathbf{d}$ is a magnified representation of predator-prey dynamics on node 5 of run 8 , where temporary local predators extinction occur

Table 2 Parameters for selected runs graphically represented in Figs. 2a, 3a and c

\begin{tabular}{|c|c|c|c|c|c|c|c|c|c|c|c|c|c|}
\hline \multicolumn{2}{|c|}{ General } & \multicolumn{9}{|c|}{ Internal species parameters } & \multicolumn{3}{|c|}{ Network metrics (global-scale) } \\
\hline run & $E$ & $n_{1}$ & $n_{2}$ & $P_{r, 1}$ & $P_{r, 2}$ & $P_{m, 2}$ & $P_{k, 2}$ & $D_{U, 1}$ & $D_{L, 1}$ & $D_{U, 2}$ & avgel & avgeg & $g c c$ \\
\hline 1 & 0 & 27 & 15 & 0.28 & 0.25 & 0.09 & 0.21 & 0.626 & 0.238 & 0.359 & 0 & 0 & 0 \\
\hline 2 & 5 & 28 & 16 & 0.3 & 0.21 & 0.1 & 0.19 & 0.793 & 0.252 & 0.4 & 0 & 0.133 & 0.3 \\
\hline 3 & 10 & 45 & 14 & 0.24 & 0.21 & 0.04 & 0.2 & 0.578 & 0.258 & 0.598 & 0.397 & 0.375 & 0.8 \\
\hline 4 & 30 & 33 & 18 & 0.28 & 0.25 & 0.06 & 0.2 & 0.701 & 0.346 & 0.3 & 0.892 & 0.833 & 1 \\
\hline 5 & 30 & 42 & 16 & 0.31 & 0.2 & 0.03 & 0.24 & 0.856 & 0.217 & 0.392 & 0.898 & 0.826 & 1 \\
\hline 6 & 35 & 46 & 18 & 0.28 & 0.31 & 0.09 & 0.12 & 0.545 & 0.349 & 0.346 & 0.934 & 0.889 & 1 \\
\hline 7 & 40 & 39 & 13 & 0.23 & 0.12 & 0.01 & 0.27 & 0.664 & 0.33 & 0.484 & 0.959 & 0.944 & 1 \\
\hline 8 & 45 & 40 & 13 & 0.34 & 0.16 & 0.08 & 0.24 & 0.58 & 0.248 & 0.475 & 1 & 1 & 1 \\
\hline
\end{tabular}

Note: 8 runs representative of the different dynamic regimes of the parameter space. This table summarizes the values of the internal species parameters and metrics of the whole network for the 8 runs

the variable dynamics stem from internal species parameters. The predation rate of predators in run 6 $\left(P_{k, 2}=0.12\right)$ is about half the value in other runs, as a result, the predators reproduce slowly due to inefficient hunting and stay at relatively low levels until sudden extinction. The early dynamics of runs 4 and 5 are almost identical; increased predation rates
$\left(P_{k, 2}=0.20\right.$ and 0.24 , respectively) means the predators are more effective at capturing prey and boosting their population. It is this prey dependency that leads to heightened predator oscillations as the efficient hunters begin to migrate from one node to another (if possible) in search of prey. The predator population in run 4 outlive that of run 5 due to the 
Table 3 Node-centrality measures for selected runs graphically represented in Figs. 2b, c, 3b, d

\begin{tabular}{|c|c|c|c|}
\hline \multicolumn{2}{|c|}{ General } & \multicolumn{2}{|c|}{ Node metrics (local-scale) } \\
\hline Run & $N_{i}$ & clos & cde \\
\hline 1 & 1 & 0 & 0 \\
\hline 1 & 5 & 0 & 0 \\
\hline 2 & 1 & 0.2 & 0.104 \\
\hline 2 & 5 & 0 & 0 \\
\hline 3 & 1 & 0 & 0 \\
\hline 3 & 5 & 0.509 & 0.457 \\
\hline 4 & 1 & 0.643 & 0.668 \\
\hline 4 & 5 & 0.9 & 0.87 \\
\hline 5 & 1 & 0.9 & 0.876 \\
\hline 5 & 5 & 0.9 & 0.876 \\
\hline 6 & 1 & 0.9 & 0.894 \\
\hline 6 & 5 & 0.75 & 0.806 \\
\hline 7 & 1 & 1 & 0.977 \\
\hline 7 & 5 & 1 & 0.977 \\
\hline 8 & 1 & 1 & 1 \\
\hline 8 & 5 & 1 & 1 \\
\hline
\end{tabular}

interplay between the movement thresholds of the prey. Note the reduced oscillations in run 4 versus run 5 on the network level in Fig. 2a. Compared to prey in run 5 , the prey population in run 4 migrate to other connected nodes when its current node is less crowded with prey or more crowded with predators, this amounts to less variability in the 'boom-bust' cycles of the predator population.

The richness in dynamics that occur on each node, and the network as a whole, do depend on the internal species parameters. Nonetheless, various network metrics allow us to draw some conclusions on the usefulness of a "corridor" or networked landscape approach. On average, well connected (or more central) patches enhance the probability of coexistence between predators and prey, independently from the different dynamics that arise across the broader parameter space analyzed.

Internal parameters such as reproduction, predation, death rates and active movement behaviour are crucial in allowing coexistence in the long-term, and give rise to different dynamic regimes as depicted in Figs. 2 and 3. Independent from internal species parameters, alteration of the landscape by connecting different patches or enhancing the centrality of particular patches still strengthens the probability of coexistence between predator and prey species (figures, parameter values, network metrics and nodecentrality measures for all the selected runs are provided in the supplemental material). In short, increasing connectivity matters, this is noticeable in run 3 of Fig. $2 b$ and c. In this run only the connectivity properties of the nodes differ, leading to quick extinction of predators on node 1 while connectivity leads to a longer persistence of predators on node 5 .

The importance of node-centrality is visualized in Fig. 4, where the behavior of average population levels for the last 1000 time-steps is evaluated based on node-centrality measures. Given the stochastic nature of the model, we smoothed the data relative to predator and prey population levels using locally weighted regression of predator and prey population on the node-centrality measures used. Additionally, we truncate the regression results so as to discard negative population levels. Basically, LOESS smoothing methods allow us to fit a low-degree polynomial regression to a subset of the observed data, giving higher weights to points nearby and lower weights to points further away from where the dependent variable is being estimated (see Cleveland 1979 and Cleveland and Devlin 1988). The weights given to distance between points of the independent variable follow the tricube weighting function, which assigns weights as follows:

$w(x)=\left\{\begin{array}{l}\left(1-|x|^{3}\right)^{3} \text { for }|x|<1 \\ 0 \text { for } \quad|x| \geq 1\end{array}\right.$

The use of smoothed data allows for a better understanding of the relationship that exists between node-centrality and population levels of predators and prey.

Spearman correlations between population levels, internal species parameters and connectivity measures have been computed (see supplementary material). Using rank-correlations allow us to further assess the important relation that exist between population levels with and the connectivity measures. Looking at node-centrality measures gives us a better understanding of local connectivity properties of a node, and consequently, the importance of that node from an ecological point of view as shown in Fig. 4, where the direct effect of measures of network connectivity on species population levels is 
visualized. Predators are the more dynamic species in this model as shown in Figs. 2, 3, and 4. In addition to the high variation in the predator population due to prey dependency and delayed growth, data collected and analysed suggests that low node/network connectivity contributes directly to predator extinction both locally and globally. For example, as supported by Fig. 4, we see that an increase in local network connectivity, as measured by closeness centrality, promotes survival and coexistence of both species. When closeness approaches 1 the population of both species begin to stabilize. A fully connected network (i.e. a network with 45 edges) represents a fully connected landscape. If a fully connected landscape, under the range of parameter values considered, promotes coexistence and convergence towards a
Fig. 4 Relation between node-centrality and population levels of predator and prey per node. Raw (the average population level of the last 1000 time-steps) and smoothed (resulted from the LOESS regressions) predator and prey population levels are reported on the $y$ axis, while node-centrality measures are represented on the $x$ axis. Graphs are drawn on the same scale
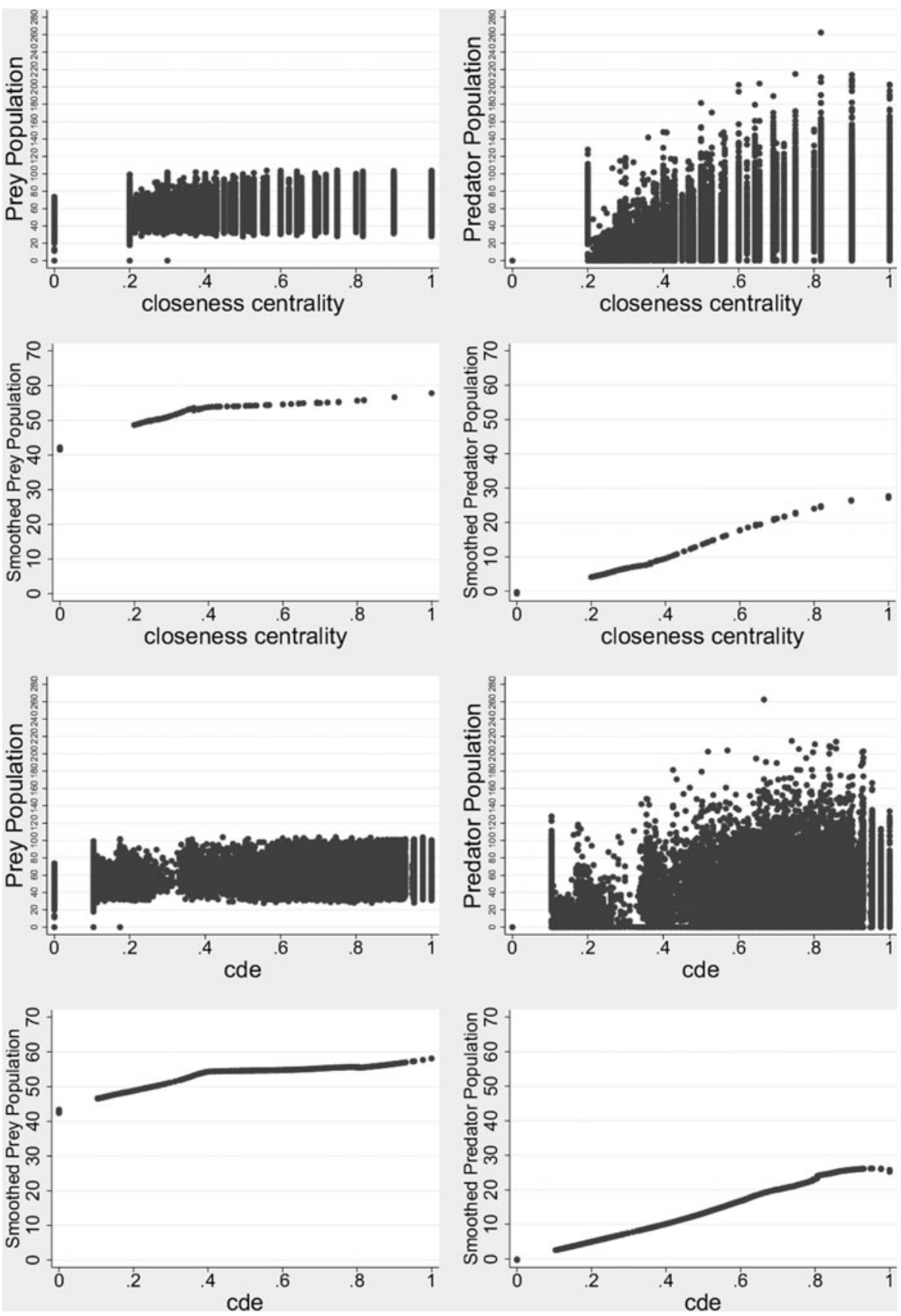
Table 4 Logit regression results

\begin{tabular}{|c|c|c|c|c|c|}
\hline \multirow[t]{2}{*}{ Indep var } & \multicolumn{2}{|l|}{ Local extinction } & \multicolumn{3}{|c|}{ Global extinction } \\
\hline & (1) & (2) & (3) & (4) & (5) \\
\hline clos & $3.505(0.023)^{*}$ & & & & \\
\hline cde & & $3.436(0.023)^{*}$ & & & \\
\hline avgel & & & $3.204(0.023)^{*}$ & & \\
\hline avgeg & & & & $3.140(0.022)^{*}$ & \\
\hline gcc & & & & & $3.910(0.029)^{*}$ \\
\hline Pseudo R & 0.179 & 0.178 & 0.157 & 0.152 & 0.167 \\
\hline Class & $67.90 \%$ & $68.79 \%$ & $67.08 \%$ & $67.49 \%$ & 67.51 \\
\hline AIC & 1.130 & 1.129 & 1.166 & 1.172 & 1.149 \\
\hline
\end{tabular}

Note $: \quad c l o s=$ closeness centrality, $c d e=(c l o s+d e g+e l) / 3$, avgel $=$ average local efficiency, avgeg $=$ global efficiency, $g c c=$ giant connected component. Standard errors in parenthesis, $*=$ significant at $1 \%$ level. 100000 observations, McFadden adjusted $R^{2}$ is reported as well as the probability of correctly classifying the dependent variable given the parameter estimates (Class). Akaike Information Criterion is reported (AIC)

viable population level (as shown in Fig. 4), is, theoretically, closeness centrality (or, more generally, node-centrality) a significant measure for assessing the probability of coexistence between species in general?

In order to answer this question, data on predator population extinction for different measures of network connectivity has been collected. Extinction of predators is recorded, at the node and network level so as to assess how node-centrality and network metrics affect the probability of predator survival. The metrics used are real numbers that take values from the closed interval $[0,1]$. Our dependent variable is a dummy variable that assumes value 0 if predators go extinct in the first 4000 time-steps on a specific patch and 1 otherwise.

The results of the logit regressions are reported in Table 4. Logit regressions have been calculated for additional node-centrality and network metrics, all of which are relevant for locally regulating dispersal of agents (i.e. all the metrics used have the same ecological meaning) (Estrada and Bodin 2008), and are reported in the supplemental material. Results are similar given the high correlation that exists between the metrics used.

Centrality measures provide a means to assess the probability of local survival of predators on a heterogeneous landscape, with closeness centrality having the strongest effect (magnitude of the coefficient) on survival probabilities as shown in Fig. 5, where predicted probabilities at specific closeness

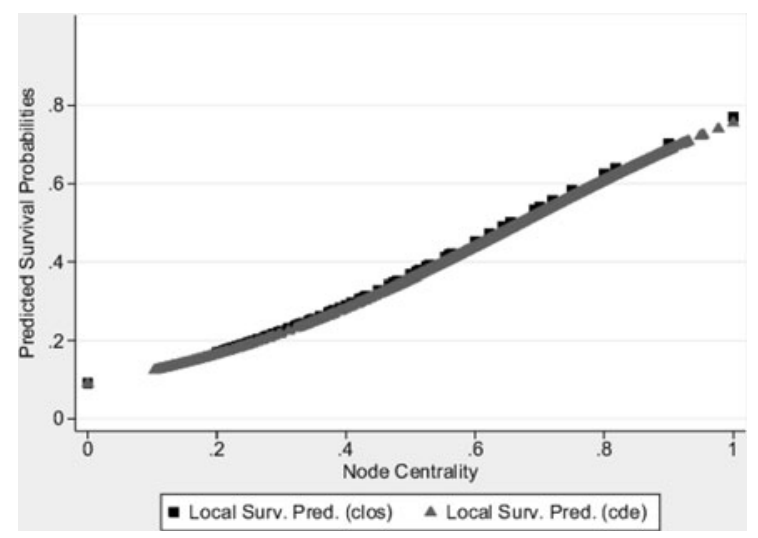

Fig. 5 Predicted predator survival probability ( $y$ axis) vs closeness centrality and cde ( $x$ axis)

centrality values and at specific cde are reported. Predator survival probabilities gradually increase depending on connectedness of the network, and no abrupt changes occur. Our results seem to indicate that even without controlling internal species parameters, enhancing the connectivity between different patches increases the probability of coexistence between predators and prey. Moreover, looking only at one patch (node) a value of closeness centrality higher than 0.65 (or a value of $c d e>0.67$ ) leads to a favourable probability $(>0.5)$ of survival.

For nodes with closeness centrality equal to zero, the probability of predator survival is low as they are unable to disperse to any other nodes in search of new prey sources and, likewise, replenishment of their 
current prey source is also impossible from outside sources. It is important to stress that a favourable probability $(>0.5)$ of survival for the predators does not guarantee species persistence. At some point, the predators may still die out, though this time to extinction grows longer as increased connectedness of the patches and heightened feasibility of migratory movement for both predators and prey decreases the odds of predator extinction. It is also important to remember that even highly connected patches do not guarantee coexistence. Internal species parameters might be of fundamental importance in allowing persistence of a species, and this is well represented by our results, which demonstrate that even in fully connected landscape networks, where centrality measures reach their maximum value, 1 , the probability predator survival is only around 0.77 .

Global survival probability is best predicted by $g c c$ (i.e. if we look at the percentage of nodes belonging to the giant connected component we have the highest probability of correctly inferring global predator survival probability), although other network metrics give similar estimates. Being connected, for a landscape network, is important as it allows species more freedom of movement and enhances their ability to migrate in search of food and safety. Global efficiency and the average local efficiency of the landscape networks examined also play an important role in assessing these probabilities. Both measures are related to the ability of the networked landscape to aid in species diffusion.

The importance of connectivity for increasing global survival probabilities is clear from the analysis performed. Both local and global survival probabilities gradually increase by increasing the level of connectivity at the node and at the network level. When the network is connected (i.e. when all its nodes belong to the giant component), global survival probabilities are enhanced, although, given the importance of internal species parameters, there is always a chance that even the most well connected network leads to some species extinction.

We assessed the robustness of our model results by conducting similar analyses in different types of networks. We first analyzed the same ABM using a random network, varying the number of edges from 0 to 45 . The results are qualitatively the same as those presented here. Next, we explored the ABM using the same "geoproximity" network but with fixed parameters at the mean values used in this study, while varying the number of edges and reconfiguring the network structure. We find similar results, although in this latter case, network centrality measures play a major role in determining predator survival probability (logit regression show a correct classification of over $90 \%$ compared to the $65 \%$ presented here). Moreover, at mean values, an increase in closeness centrality from 0 to 0.2 leads to an increase in predator survival probability from 0.09 to $5.79 \%$, while an increase of closeness centrality from 0.2 to 0.4 leads to an increase of survival probability from 5.79 to $80.66 \%$, thus suggesting the existence of "connectivity thresholds" for species with certain reproduction, death and predation rates. Lastly, using mean values, favourable survival probabilities (i.e. $>0.5$ ) arise at a much lower level of closeness centrality (0.333 instead of 0.65 ).

\section{Discussion}

Understanding the ecological consequences of habitat fragmentation is becoming increasingly important, given the mounting pressure of human population and the possible effects of climatic changes. Addressing possible effects of fragmentation on predator-prey dynamics becomes crucial for biodiversity conservation. In this context, our study highlights three main findings. First, at high levels of node-centrality for different patches, the predators move so rapidly through the fully connected network that low prey numbers in any specific node do not hamper their population growth. In other words, predators become a successful population of migrants constantly moving in search of patches where it is easier to find, attack, and feed on prey. Second, increasing connectivity benefits both predator and prey species however, the maximum population for both species occurs at different levels of connectivity. Third, the results from the ABM match theoretical expectations from corridor ecology (Hilty et al. 2006).

The first finding demonstrates that the connectivity of a landscape gains importance to predators as they become increasingly dependent on movement through different patches (ecological areas) due to range requirements (Coppolillo et al. 2004). Jaguars present a real world example of predators with large ranges that are highly dependent on movement 
between different patches, as landscape connectivity is essential for their survival (Ortega-Huerta and Medley 1999; Michalski and Peres 2005). Loss of prey is the driving force that leads predators to move between patches in the model presented here, and the possibility of migration is a key aspect for their survival.

The second finding is that both predator and prey species benefit from living in globally central patches (i.e. with high closeness centrality) as shown in Fig. 4. However, the maximum population of prey is reached, on average, at lower closeness centrality levels compared to predators. Prey benefit from the constraints imposed on predator movement since they can enhance their number as the risk of predation and the use of anti-predatory strategies decreases. The reintroduction of gray wolves in North America and the resultant decline in elk population densities provides a real world example of our model results. Increasing connectivity, and living on globally central and locally central patches, enhances the probability of coexistence between prey and predators. In this instance the 'top-down' process of predation keeps prey populations in check while allowing for coexistence which is fundamental for the maintenance of biodiversity (Terborgh et al. 1999).

Additionally, the study presented confirms the general theory behind the corridor ecology approach (Hilty et al. 2006) as stated above. Here, conservation centers upon connecting different patches rather than constructing isolated protected areas or islands of conservation. Moreover, it is important to notice how internal species parameters, such as reproduction rates, death rates, predation rates and active decision regarding migration, have a significant effect upon population levels. However, while human management of the connectivity between different patches is possible, altering species internal parameter on a large scale is not (at least with present-day technology).

To conclude, the results of the effect of increasing connectedness between patches on predator-prey dynamics can lead to different ways to manage a given landscape. Although we recognize that a simple network may hide important variables that drive predator-prey dynamics in reality, our simple network representation still allows for a coarse-grained assessment of which management strategies lead to set managerial goals. We are fully aware that strategies of managing landscapes differ according to the management objective, and further research is currently underway to explore possible landscape management strategies, where predators and prey dynamically interact.

Acknowledgements We thank the two anonymous referees as well as the financial support of Arizona State University to facilitate the first author to visit ASU during the first half of 2009.

\section{References}

Benson DL, Sherratt JA, Maini PK (1993) Diffusion driven instability in an inhomogeneous domain. Bull Math Biol 55(2):365-384

Blasius B, Huppert A, Stone L (1999) Complex dynamics and phase synchronization in spatially extended ecological systems. Nature 399:356-359

Boccaletti S, Latora V, Moreno Y, Chavez M, Hwang D-H (2006) Complex networks: structure and dynamics. Phys Rep 424(4-5):175-308

Bodin Ö, Norberg J (2007) A network approach for analyzing spatially structured populations in fragmented landscape. Landscape Ecol 22(1):31-44

Bolker BM (2003) Combining endogenous and exogenous spatial variability in analytical population models. Theor Popul Biol 64(3):255-270

Breckling B, Middelhoff U, Reutera H (2006) Individual-based models as tools for ecological theory and application: Understanding the emergence of organisational properties in ecological systems. Ecol Modell 194(1-3):102-113

Cleveland WS (1979) Robust locally weighted regression and smoothing scatterplots. J Am Stat Assoc 74(368):829-836

Cleveland WS, Devlin SJ (1988) Locally weighted regression: an approach to regression analysis by local fitting. J Am Stat Assoc 83(403):569-610

Cominsa HN, Hassell MP (1996) Persistence of multispecies host-parasitoid interactions in spatially distributed models with local dispersal. J Theor Biol 183(1):19-28

Coppolillo P, Gomez H, Maisels F, Wallace R (2004) Selection criteria for suites of landscape species as a basis for sitebased conservation. Biol Conserv 115(3):419-430

Creel S, Winnie J Jr, Maxwell B, Hamlin K, Creel M (2005) Elk alter habitat selection as an antipredator response to wolves. Ecology 86(12):3387-3397

Crucitti P, Latora V, Marchiori M, Rapisarda A (2004) Error and attack tolerance of complex networks. Physica A 340(1-3):388-394

Cuddington KM, Yodzis P (2000) Diffusion-limited predatorprey dynamics in euclidean environments: an allometric individual-based model. Theor Popul Biol 58(4):259-278

da Fontoura Costa L, Rodrigues FA, Travieso G, Villas Boas PR (2007) Characterization of complex networks: a survey of measurements. Adv Phys 56(1-2):167-242

DeAngelis DL, Mooij WM (2005) Individual-based modeling of ecological and evolutionary processes. Annu Rev Ecol Evol Syst 36:147-168 
Droz M, Pekalski A (2001) Coexistence in a predator-prey system. Phys Rev E 63(5), 051909.051901-051909. 051906

Estrada E, Bodin Ö (2008) Using network centrality measures to manage landscape connectivity. Ecol Appl 18(7):18101825

Fahrig L (1998) When does fragmentation of breeding habitat affect population survival? Ecol Modell 105(2-3):273292

Fahrig L, Nuttle WK (2005) Population ecology in spatially heterogeneous environments. In: Lovett GM, Turner MG, Jones CG, Weathers KC (eds) Ecosystem function in heterogeneous landscapes. Springer, New York

Fischhoff IR, Sundaresan SR, Cordingley J, Rubenstein DI (2007) Habitat use and movements of plains zebra (Equus burchelli) in response to predation danger from lions. Behav Ecol 18(4):725-729

Grimm V, Railsback SF (2005) Individual-based modeling and ecology. Princeton University Press, Princeton, NJ

Grimm V, Berger U, Bastiansen F, Eliassen S, Ginot V, Giske J, Goss-Custard J, Grand T, Heinz SK, Huse G, Huth A, Jepsen JU, Jørgensen C, Mooij WM, Müller B, Pe'er G, Piou C, Railsback SF, Robbins AM, Robbins MM, Rossmanith E, Rüger N, Strand E, Souissi S, Stillman RA, Vabø R, Visser U, DeAngelis DL (2006) A standard protocol for describing individual-based and agent-based models. Ecol Modell 198(1-2):115-126

Hastings A (2001) Transient dynamics and persistence of ecological systems. Ecol Lett 4(3):215-220

Hilty JA, Lidicker WZ Jr, Merenlender AM (2006) Corridor ecology: the science and practice of linking landscapes for biodiversity conservation. Island Press, Washington, DC

Holland MD, Hastings A (2008) Strong effect of dispersal network structure on ecological dynamics. Nature 456(7395):792-794

Hovel KA, Regan HM (2008) Using an individual-based model to examine the roles of habitat fragmentation and behavior on predator-prey relationships in seagrass landscapes. Landscape Ecol 23(Suppl 1):75-89

Inchausti P, Ballesteros S (2008) Intuition, functional responses and the formulation of predator-prey models when there is a large disparity in the spatial domains of the interacting species. J Anim Ecol 77(5):891-897

Ioannou CC, Ruxton GD, Krause J (2008) Search rate, attack probability, and the relationship between prey density and prey encounter rate. Behav Ecol 19(4):842-846

Ives AR, Dobson AP (1987) Antipredator behavior and the population dynamics of simple predator-prey systems. Am Nat 130(3):431-447

Jansen VAA (2001) The dynamics of two diffusively coupled predator-prey populations. Theor Popul Biol 59(2):119131

Kareiva P (1987) Habitat fragmentation and the stability of predator-prey interactions. Nature 326:388-390

Latora V, Marchiori M (2001) Efficient behavior of smallworld networks. Phys Rev Lett 87(19), 198701-198701 198701-198704

Lima SL (2002) Putting predators back into behavioral predator-prey interactions. Trends Ecol Evol 17(2):70-75

Linhares A (1999) Synthesizing a predatory search strategy for VLSI layouts. IEEE Trans Evol Comput 3(2):147-152
Luttberg B, Schmitz OJ (2000) Predator and prey models with flexible individual behavior and imperfect information. Am Nat 155(5):669-683

McCauley E, Wilson WG, de Roos AM (1993) Dynamics of age-structured and spatially structured predator-prey interactions: individual-based models and populationlevel formulations. Am Nat 142(3):412-442

McLaughlin JF, Roughgarden JD (1991) Pattern and stability in predator-prey communities: how diffusion in spatially variable environments affects the Lotka-Volterra model. Theor Popul Biol 40(2):148-172

Michalski F, Peres CA (2005) Anthropogenic determinants of primate and carnivore local extinctions in a fragmented forest landscape of southern Amazonia. Biol Conserv 124(3):383-396

Minor ES, Urban DL (2007) Graph theory as a proxy for spatially explicit population models in conservation planning. Ecol Appl 17(6):1771-1782

Minor ES, Urban DL (2008) A graph-theory framework for evaluating landscape connectivity and conservation planning. Conserv Biol 22(2):297-307

Nelson EH, Matthews CE, Rosenheim JA (2004) Predators reduce prey population growth by inducing changes in prey behavior. Ecology 85(7):1853-1858

Nonaka E, Holme P (2007) Agent-based model approach to optimal foraging in heterogeneous landscapes: effects of patch clumpiness. Ecography 30(6):777-788

Ortega-Huerta MA, Medley KE (1999) Landscape analysis of jaguar (Panthera onca) habitat using sighting records in the Sierra de Tamaulipas, Mexico. Environ Conserv 26(4):257-269

Pascual-Hortal L, Saura S (2006) Comparison and development of new graph-based landscape connectivity indices: towards the priorization of habitat patches and corridors for conservation. Landscape Ecol 21(7):959-967

Planesa S, Jones GP, Thorrold SR (2009) Larval dispersal connects fish populations in a network of marine protected areas. PNAS (0808007106v1-pnas.0808007106)

Rougharden JD (1977) Patchiness in the spatial distribution of a population caused by stochastic fluctuations in resources. Oikos 29(1):52-59

Rougharden JD (1978) Influence of competition on patchiness in a random environment. Theor Popul Biol 14(2):185203

Terborgh J, Estes JA, Paquet P, Ralls K, Boyd-Heger D, Miller BJ, Noss RF (1999) The role of top carnivores in regulating terrestrial ecosystems. In: Soule ME, Terborgh J (eds) Continental conservation: scientific foundations of regional reserve networks. Island Press, Washington, DC

Urban D, Keitt T (2001) Landscape connectivity: a graphtheoretic perspective. Ecology 82(5):1205-1218

Urban DL, Minor ES, Treml EA, Schick R (2009) Graph models of habitat mosaics. Ecol Lett 12(3):260-273

Wilson WG (1998) Resolving discrepancies between deterministic population models and individual-based simulations. Am Nat 151(2):116-134

With KA, Pavuk DM, Worchuck JL, Oates RK, Fisher JL (2002) Threshold effects of landscape structure on biological control in agroecosystems. Ecol Appl 12(1):52-65 